\title{
Optimizing FDG-PET/CT imaging of inflammation in atherosclerosis
}

\author{
Antti Saraste, $M D, P h D,{ }^{a, b}$ and Juhani Knuuti, $M D, P h D^{a}$ \\ ${ }^{\text {a }}$ Turku PET Centre, Turku University Hospital and University of Turku, Turku, Finland \\ ${ }^{\mathrm{b}}$ Turku Heart Center, Turku University Hospital and University of Turku, Turku, Finland
}

Received Feb 26, 2015; accepted Feb 26, 2015

doi: 10.1007/s12350-015-0112-9

\section{See related article, pp. 468-479}

\section{INTRODUCTION}

Positron emission tomography with 18Ffluorodeoxyglucose (18F-FDG-PET) has been shown to detect metabolic activity as a marker of local inflammation of the arterial wall or atherosclerotic plaque. Inflammation is a key factor in the pathogenesis of atherosclerosis and atherosclerotic plaque rupture underlying many of its complications. ${ }^{1}$ Significant correlations between 18F-FDG-PET vascular uptake and macrophage content in the atherosclerotic plaque, the presence of cardiovascular risk factors, systemic markers of inflammation, and high-risk anatomical or histological plaque features support the role of $18 \mathrm{~F}$ FDG-PET as a prognostic biomarker. ${ }^{2,3}$ Furthermore, retrospective analyses of PET images from patients who had 18F-FDG-PET for cancer imaging suggest that enhanced arterial 18F-FDG uptake is associated with future risk of cardiovascular disease (CVD) events. ${ }^{4,5}$ To better understand the prognostic value of 18F-FDGPET, the results of the prospective studies are awaited that will provide cardiovascular outcome data for asymptomatic individuals at high cardiovascular risk who underwent carotid and aortic 18F-FDG-PET. ${ }^{6}$ Already now, 18F-FDG-PET has been used in several clinical trials of novel anti-atherosclerotic therapies as a surrogate marker of treatment efficacy. ${ }^{2,3}$ It has potential to show the positive effect of therapy in a small patient population that can serve as a proof-of-concept for larger clinical trials based on clinical outcomes. For

Reprint requests: Antti Saraste MD, PhD, Turku PET Centre, Turku University Hospital and University of Turku, Kiinamyllynkatu 4-8, 20520 Turku, Finland; antti.saraste@utu.fi

J Nucl Cardiol 2015;22:480-2.

$1071-3581 / \$ 34.00$

Copyright (c) 2015 American Society of Nuclear Cardiology. wide-spread clinical application and correct interpretation of findings, it is essential that the results of $18 \mathrm{~F}$ FDG-PET are reproducible and comparable among different trials and institutions. Therefore, standardization of imaging protocols and data analysis is important.

To measure 18F-FDG-PET signal, circular regions of interest (ROI) are drawn around the artery in axial cross sections to determine mean and maximum standardized uptake values (SUV). Most often, tissue-tobackground ratio (TBR), which may be considered to best reflect the vessel wall uptake, is reported by correcting the arterial SUV with SUV for blood pool activity in the superior vena cava. Validation studies have demonstrated that 18F-FDG-PET of carotid, aortic, and peripheral arteries is highly reproducible over 2 weeks period in asymptomatic patients with documented CVD or risk factors for CVD. ${ }^{7,8}$ Based on these results and analysis of previous literature, Rudd et al made recommendations for standardization of $18 \mathrm{~F}$ FDG-PET vascular imaging in 2008. ${ }^{8}$ It was advised that the use of combined PET/CT scanners is desirable to facilitate accurate co-registration of the PET and CT images of the arteries. For arterial imaging, a longer circulation time than for cancer imaging, preferably at least 90 minutes, was recommended. This allows sufficient $18 \mathrm{~F}-\mathrm{FDG}$ to accumulate in the arterial wall where $18 \mathrm{~F}-\mathrm{FDG}$ becomes permanently trapped and to permit background levels of 18F-FDG in the blood to become reduced by as much as possible. Since then, 18F-FDGPET has been used in many clinical trials, and new methodological studies have been published.

In this issue of the journal, Gholami et al address the strengths and weaknesses of the various imaging protocols and measurement strategies used in arterial 18F-FDG-PET based on the recent literature. ${ }^{9}$ They note that high 18F-FDG dose is not necessary to obtain sufficient image quality and accurate lesion quantification that is important to optimize the radiation dose to the patient. In contrast, timing of imaging after tracer injection has significant effect on arterial 18F-FDG signal. Optimal circulation time of 18F-FDG for arterial 
imaging may be longer than 90 minutes, but authors note that more comparative studies are needed to define it. $^{9-11}$ Similarly, even moderate hyperglycaemia can lower 18 F-FDG uptake by plaque cells. ${ }^{10}$ This is probably mainly based on competition between glucose and $18 \mathrm{~F}$ FDG as metabolic substrates and more tracer remains in circulation. Therefore, it may be advisable to avoid fasting glucose levels above $7 \mathrm{mmol} \cdot \mathrm{L}(126 \mathrm{mg} \cdot \mathrm{dL}) .^{9,10}$ Diet interventions can efficiently reduce physiological glucose uptake by the myocardium that is essential for 18F-FDG imaging of coronary arteries. ${ }^{12}$ However, as Gholami et al note more comparative studies on the effects of different diet interventions and pre-scan glucose levels on the atherosclerotic uptake of 18F-FDG would be still needed.

Different approaches have been applied for measurement of arterial 18F-FDG uptake in different studies as described by Gholami et al. ${ }^{9}$ 18F-FDG uptake values from multiple sections can be averaged within an entire artery or arteries. This can offer an accurate estimate of overall plaque activity and may be best suited for monitoring the response to systemic treat-ments for atherosclerosis. On the other hand, measuring 18F-FDG uptake in a selected arterial segment is probably optimal for detecting and monitoring the effects of local, plaquebased therapy. Furthermore, selection of the most active or diseased segments for analysis may be preferred because they may be most likely to show positive treatment effects due to the presence of active inflammation or atherosclerosis. Selection of the artery or segments for analysis (i.e., index vessel or most diseased segments) can be based on 18F-FDG uptake at baseline or at follow-up as well as the presence of morphological evidence of atherosclerosis in CT or MRI images. A source of variation is that a generally accepted vesselspecific threshold for abnormal 18F-FDG signal in atherosclerosis has not been defined yet. ${ }^{9}$ Relatively small studies with methodological differences have compared in vivo $18 \mathrm{~F}-\mathrm{FDG}$ signal with the histological basis of $18 \mathrm{~F}-\mathrm{FDG}$ uptake in atherosclerotic plaques. ${ }^{13}$ Furthermore, it may be that $18 \mathrm{~F}-\mathrm{FDG}$ uptake is present only transiently during the natural course of atherosclerotic plaque development. ${ }^{14,15}$ Authors conclude that there is a need for studies aiming to find out which of the $18 \mathrm{~F}-\mathrm{FDG}$ analysis methods best reflect the true value of 18 F-FDG uptake in the vessel wall and histological correlates of inflammation.

Coronary arteries are challenging targets for $18 \mathrm{~F}$ FDG-PET due to small size, cardiac, and respiratory motion in images and high physiological tracer uptake in the adjacent myocardium. However, evaluation of coronary arteries is important because large proportion CVD events are related to coronary artery disease. Imaging small structures, such as atherosclerotic plaques, with PET is challenging owing to partial volume and scatter effects given the low spatial resolution of the technique. Measured SUV might not, therefore, reflect true tracer uptake within the lesion. However, studies have not so far demonstrated superiority of partial volume-corrected arterial 18F-FDG quantification over non-corrected in the carotid arteries imaged by both PET and magnetic resonance imaging (MRI). ${ }^{13}$ Proof-of-concept studies have shown the potential feasibility of detecting $18 \mathrm{~F}$ FDG accumulation in the coronary arteries. ${ }^{3} 18 \mathrm{~F}-\mathrm{FDG}$ signal was shown to be higher in the culprit lesions of acute coronary syndromes (unstable angina or acute myocardial infarction) than in stable coronary atherosclerotic plaques. ${ }^{16}$ There are ongoing efforts to improve 18F-FDG-PET of coronary plaques, such as application of diet interventions to suppress myocardial glucose uptake ${ }^{12}$ and motion correction using respiratory and ECG signals. ${ }^{17}$ This should open possibilities to study how well 18F-FDG uptake in the coronaries and peripheral arteries correlates with each other, and thus whether peripheral artery 18F-FDG-PET is appropriate as a surrogate of coronary artery inflammation.

Novel tracers applied to atherosclerosis imaging might improve the specificity of the PET inflammatory signal and help to overcome obstacles complicating coronary artery imaging. ${ }^{1,2}$ Initial reports have described successful visualization of $18 \mathrm{~F}-\mathrm{NaF}$ reflecting active calcification in the coronary arteries of patients with acute coronary syndromes. ${ }^{18}$ Other potential tracers are being tested humans, such as those targeting macrophage receptors ${ }^{1,2,19}$ or neovascularization. ${ }^{20}$ Arterial 18F-FDG is a promising technique, but as highlighted in the article of Gholami et al, information about basic protocols and signal analysis is needed for wide-spread application of molecular imaging of atherosclerosis, especially in the coronary arteries.

\section{Acknowledgments}

The authors acknowledge financial support from The Academy of Finland Centre of Excellence on Cardiovascular and Metabolic Diseases, Helsinki, Finland and Finnish Foundation for Cardiovascular Research.

\section{References}

1. Tavakoli S, Vashist A, Sadeghi MM. Molecular imaging of plaque vulnerability. J Nucl Cardiol 2014;21:1112-28.

2. Tarkin JM, Joshi FR, Rudd JH. PET imaging of inflammation in atherosclerosis. Nat Rev Cardiol 2014;11:443-57.

3. Rudd JH, Narula J, Strauss HW, Virmani R, Machac J, Klimas M, et al. Imaging atherosclerotic plaque inflammation by fluorodeoxyglucose with positron emission tomography: Ready for prime time? J Am Coll Cardiol 2010;55:2527-35.

4. Rominger A, Saam T, Wolpers S, Cyran CC, Schmidt M, Foerster $\mathrm{S}$, et al. 18F-FDG PET/CT identifies patients at risk for future 
vascular events in an otherwise asymptomatic cohort with neoplastic disease. J Nucl Med 2009;50:1611-20.

5. Figueroa AL, Abdelbaky A, Truong QA, Corsini E, MacNabb MH, Lavender ZR, et al. Measurement of arterial activity on routine FDG PET/CT images improves prediction of risk of future CV events. JACC Cardiovasc Imaging 2013;6:1250-9.

6. Muntendam P, McCall C, Sanz J, Falk E, Fuster V. The BioImage Study: Novel approaches to risk assessment in the primary prevention of atherosclerotic cardiovascular disease-study design and objectives. Am Heart J 2010;160:49-57.

7. Rudd JH, Myers KS, Bansilal S, Machac J, Rafique A, Farkouh M, et al. (18)Fluorodeoxyglucose positron emission tomography imaging of atherosclerotic plaque inflammation is highly reproducible: Implications for atherosclerosis therapy trials. J Am Coll Cardiol 2007;50:892-6.

8. Rudd JH, Myers KS, Bansilal S, Machac J, Pinto CA, Tong C, et al. Atherosclerosis inflammation imaging with 18F-FDG PET: Carotid, iliac, and femoral uptake reproducibility, quantification methods, and recommendations. J Nucl Med 2008;49:871-8.

9. Gholami S, Salavati A, Houshmand S, Werner TJ, Alavi A. Assessment of atherosclerosis in large vessel walls: A comprehensive review of FDG-PET/CT image acquisition protocols and methods for uptake quantification. J Nucl Cardiol 2015, in press.

10. Bucerius J, Mani V, Moncrieff C, Machac J, Fuster V, Farkouh M. EOptimizing 18F-FDG PET/CT imaging of vessel wall inflammation: The impact of 18F-FDG circulation time, injected dose, uptake parameters, and fasting blood glucose levels. Eur J Nucl Med Mol Imaging 2013;41:369-83.

11. Blomberg BA, Thomassen A, Takx RA, Hildebrandt MG, Simonsen JA, Buch-Olsen KM, et al. Delayed ${ }^{18}$ F-fluorodeoxyglucose PET/CT imaging improves quantitation of atherosclerotic plaque inflammation: Results from the CAMONA study. J Nucl Cardiol 2014;21:588-97.
12. Demeure F, Hanin FX, Bol A, Vincent MF, Pouleur AC, Gerber B, et al. A randomized trial on the optimization of 18F-FDG myocardial uptake suppression: Implications for vulnerable coronary plaque imaging. J Nucl Med 2014;55:1629-35.

13. Izquierdo-Garcia D, Davies JR, Graves MJ, Rudd JH, Gillard JH, Weissberg PL, et al. Comparison of methods for magnetic resonanceguided [18-F]fluorodeoxyglucose positron emission tomography in human carotid arteries: Reproducibility, partial volume correction, and correlation between methods. Stroke 2009;40:86-93.

14. Wassélius J, Larsson S, Jacobsson H. Time-to-time correlation of high-risk atherosclerotic lesions identified with [(18)F]-FDG-PET/ CT. Ann Nucl Med 2009;23:59-64.

15. Ben-Haim S, Kupzov E, Tamir A, Frenkel A, Israel O. Changing patterns of abnormal vascular wall F-18 fluorodeoxyglucose uptake on follow-up PET/CT studies. J Nucl Cardiol 2006;13:791-800.

16. Rogers IS, Nasir K, Figueroa AL, Cury RC, Hoffmann U, Vermylen DA, et al. Feasibility of FDG imaging of the coronary arteries: Comparison between acute coronary syndrome and stable angina. JACC Cardiovasc Imaging 2010;3:388-97.

17. Teräs M, Kokki T, Durand-Schaefer N, Noponen T, Pietilä M, Kiss J, et al. Dual-gated cardiac PET-clinical feasibility study. Eur J Nucl Med Mol Imaging 2010;37:505-16.

18. Joshi NV, Vesey AT, Williams MC, Shah AS, Calvert PA, Craighead $\mathrm{FH}$, et al. (18)F-fluoride positron emission tomography for identification of ruptured and high-risk coronary atherosclerotic plaques: A prospective clinical trial. Lancet 2014;383:705-13.

19. Rominger A, Saam T, Vogl E, Ubleis C, la Fougère C, Förster S, et al. In vivo imaging of macrophage activity in the coronary arteries using 68 Ga-DOTATATE PET/CT: Correlation with coronary calcium burden and risk factors. J Nucl Med 2010;51:193-7.

20. Beer AJ, Pelisek J, Heider P, Saraste A, Reeps C, Metz S, et al. PET/CT imaging of integrin $\alpha \mathrm{v} \beta 3$ expression in human carotid atherosclerosis. JACC Cardiovasc Imaging 2014;7:178-87. 